International Journal of Systems Signal Control and Engineering Application 12 (1): 13-20, 2019

ISSN: 1997-5422

(C) Medwell Journals, 2019

\title{
An Efficient Technique of QRS Complex Detection of Electrocardiography Signal Based on Optimized Median Filter and Efficient Signal Reshaping
}

\author{
Raghad Wahab Mohammed Haider J. Abd \\ Department of Electrical, College of Engineering, University of Babylon, Karbala, Iraq
}

\begin{abstract}
Automatic detection of R-peak is changing into a prominent tool for automatizing the designation of significant cardiovascular components. R-wave peaks in electrocardiography is a major indicator of the cardiac dysfunction. Most of the R-wave peaks prospectors have great troubles owing to non-stationary attitude of the ECG signal. In our current research, a smart technique established on bases of median filter and an automated R-wave peaks detection technique has been proposed. First median filter approach is used to eliminate the base line from the ECG signal. Then an efficient signal reshaping with sequential steps was proposed to detect the QRS position. The efficiency of the proposed work has been tested on MIT-BIH arrhythmia database. The technique has also been applied to a real signal for patients with different heart diseases. The proposed technique showed better performance and faster detection compared to the latter techniques at the same time. Error rate detection is low $0.05 \%$, Positive predictivity $(\mathrm{P}+$ ) of $99.85 \%$ Sensitivity (Se) of $99.94 \%$ and an average F-score of 0.999 are accomplished for the suggested ECG detector which are superior to the former results.
\end{abstract}

Key words: ECG signal, median filter, R-peak, MIT-BIH database, signal reshaping

\section{INTRODUCTION}

In recent years Associate in nursing calculable 17.3 million individuals have died of cardiovascular disease (WHO., 2011), representing thirteen of the world's deaths. The primary reason for death worldwide is disorder in line with the globe health organization. Medical interference and technology have evolved attributable to the nice importance that medical analyzers have placed on heart health research (Dilaveris et al., 1998; Pan and Tompkins, 1985; Silva et al., 2011). Electrocardiogram has been a straightforward, cheap and common-use check electrocardiograph that show the heart's health, it contributes to the diagnosing of the many heart. Subsequently, the analysis of electrocardiogram signals was explained during 20 years ago (Dilaveris et al., 1998; Pan and Tompkins, 1985; Silva et al., 2011; El-Dahshan, 2011).

Electrocardiogram (ECG) is one of the important factors for diagnosing cardiac illnesses. It presents the electrical activities of the heart. The primary segments of a typical ECG signal are P-wave, QRS complex and T-wave. The $P$ wave presents the contraction of the atrial rooms of the heart, the QRS complex exhibits the contraction of the ventricular rooms and finally the $T$ wave is the relaxation of the ventricular rooms, Table 1 shows the abbreviations of ECG beats with their definitions.

The main target of this study is to introduce an efficient algorithm for the detection of the QRS complex
Table 1: ECG beats abbreviations

\begin{tabular}{ll}
\hline Terms & Abbreviations \\
\hline Electrocardiography & ECG \\
Unclassifiable beat & $\mathrm{Q}$ \\
Right bundle branch block beat & $\mathrm{R}$ \\
Supraventricular premature beat & $\mathrm{S}$ \\
Electrocardiography waves & $\mathrm{P}, \mathrm{QRS}, \mathrm{T}$ and U \\
Ventricular trigeminy & $\mathrm{T}$ \\
Premature Ventricular Complex & $\mathrm{PVC}$ \\
Atrial Premature Contraction & $\mathrm{APC}$ \\
Electrocardiography & EKG \\
Electromyography & EMG \\
Left Bundle Branch Block & LBBB \\
Right Bundle Branch Block & RBBB \\
\hline
\end{tabular}

at ECG signals based on median filter and optimized signal processing. This algorithm aims to improve the performance of QRS detection

Literature review: There are several QRS complex detection strategies sophisticated by researchers within the last 3 decades with utilization of many techniques which includes: derivatives, Yeh and Wang (2008) and Arzeno et al. (2008), digital filters (Manikandan and Soman, 2012; Hamilton and Tompkins, 1986; Adnane et al., 2009), wavelet-transform (Sahambi et al., 1997; Saxena et al., 2002; Martinez et al., 2004; Ghaffari et al., 2008, 2009; Sunkaria et al., 2010; Chouakri et al., 2011), neural networks (Vijaya et al., 1997), Support Vector Machine (SVM) (Mehta and Lingayat, 2008), mathematical morphology (Zhang and Lian, 2009), combined threshold procedure (Christov, 2004), moving averaging technique (Chen et al., 2006), 
phase space process (Plesnik et al., 2012), Hilbert transform procedure (Benitez et al., 2000) and body sensor which is based on a network ( $\mathrm{Li}$ and Tan, 2006). By assuming a noise-free ECG signal, the derivative-based algorithms and digital filters calculate QRS complex without the need for the $\mathrm{P}$ and $\mathrm{T}$ waves were filtered by a low, high and band pass filters. In other algorithms it is assumed that there is a specific frequency range in which there are predefined QRS complexes as in wavelet transform algorithms utilize a collection of low path and high path filter (Zidelmal et al., 2014).

There are computationally complex methods that require extensive training and good estimation of model parameters as well as more pre-processing steps as in the algorithms based on Artificial Neural Network (ANN) and SVM. However, these techniques for QRS detection are difficult in the application and needs almost costly.

A new simple method to detect QRS has been suggested in this study, based on optimization median filter and signal re-shaping. The results showed the superiority of the proposed technology compared to other technologies in the same conditions where the parameters were tested through the MIT-BIH database for accurate justification.

Theoretical backgrounds: Generally, in ECG signal the QRS complex detection are often divided in 2 main sections: the primary one is elimination of the noise and second is QRS complex revelation. Electrocardiogram signal recording possess a noise of 50/60 Hz Power Line Interference (PLI) and because of that, also muscular quiver which will create EMG noise that belong to high frequency noise, also because of unexpected subject movement or motion artifact owing to poor electrode fitting or respiration are going to be composed wander baseline drift.

The Wander baseline drift and motion drift comes from an occasional frequency wherever the frequency within the wander baseline drift contained a smaller amount than $1 \mathrm{~Hz}$. During our work, wander baseline drift intended only to be removable and the QRS complex is determined in the existence of noises substitutional.

Within this research in order to eliminate wander baseline drift, we tend to thought of a non- recursive median filter. The median filter could be a technique of a nonlinear digital filtering, typically want to remove noises from associate signal or image (Huang, 1981).

Pre-processing is a typical step to reduce noise and to enhance the results of later process. Median filter below certain conditions utilized in digital image processing as a result of preserves edges whereas removing noise and conjointly utilized in applications signal processing. It will be wide used. The propose QRS complex detection technique utilizes an easy two stages median filter to

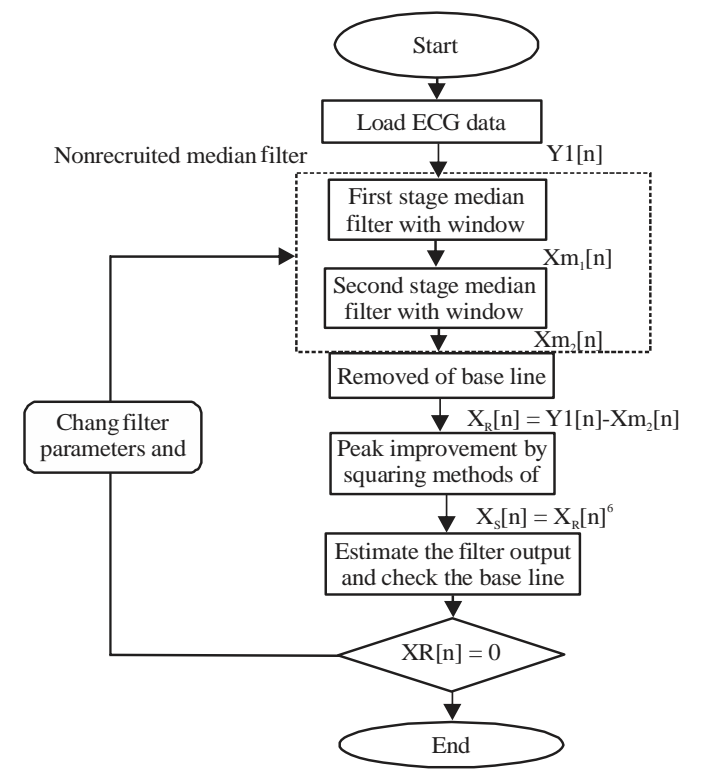

Fig. 1: Flowchart describes of median filter

eliminate baseline drift via. utilizing 2 window widths considering sampling frequency of registered datum. Then, through the multiplication of the data (extracted from base line signal which is noise free) six point-to-point times wherever sharp peaks like a Q, R, S area unit increased over artifacts and P \& T waves. Once power of the signal is become sixth, mean values of signal become above $\mathrm{P}$ and $\mathrm{T}$-waves and every one artifact. The norm of signals are clearly, especially, waves except QRS waves.

The detail step of preprocessing is explained within the flowchart Fig. 1. For detection QRS automatic, a threshold value is needed to differentiate between alternative ECG wave like P and T waves and QRS complex components. The cut point is expounded to extend of top extent of QRS complex value.

\section{MATERIALS AND METHODS}

Proposed system diagram of QRS detection: A graphical diagram of the QRS complex detection proposed method is appearing in Fig. 2. Generally, the detection method will be divided into five stages, the perform of every step is expound as follows:

Median filter: The central idea of the median filter is to have the input signal by input and replace each entry with the median of the adjacententries (Dohare et al., 2014). The style of neighbors named the "window" that slide, entry by entry, along the entire signal. For one-Dimensional (1D) signals, the chief window evident is the primary few preceding and following entries, meanwhile two-Dimensional (2D) or higher-dimensional 
Int. J. Syst. Signal Control Eng. Appl., 12 (1): 13-20, 2019

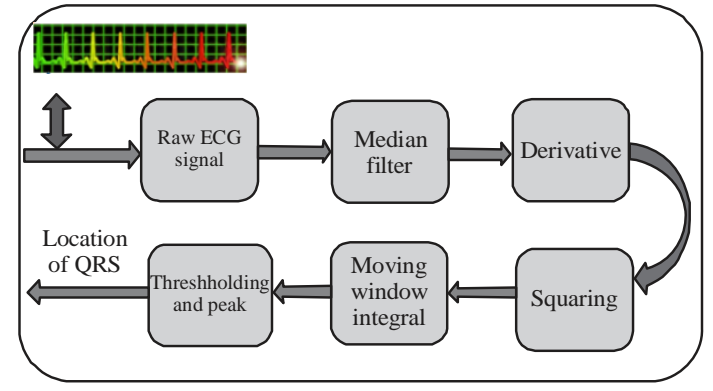

Fig. 2: Schematic diagram of the proposed QRS detection technique

signals like images, themost sophisticated window are designs are doable. It's important to notify that if the windows have entries with odd values, the median is estimated with ease and simply undertaking the entries through investigated window numerically. In order to obtain subtle range of entries, there are more than 1 possible median. The output of non-recursive filter at some extent is that the median value of the input data within the window focused at the point. If " $x(k) \leq k \geq L$ \} and $y(k) \leq k \geq L$ ?", severally, the input and output of the one-Dimensional (1-D) SM filter of window size $2 \mathrm{~N}+1$, then:

$$
y(k)=\operatorname{med}\{x(k-N), \ldots, x(k-1), x(k), x(k+1), \ldots, x(k+N)\}
$$

For account start and final effect, $x(L)$ and $x(1)$, respectively are repeated $\mathrm{N}$ times at the start and at the finish of the input.

Derivative: After the median filter processing, for the QRS advanced inclination, the free of noise cardiogram signal is gives to the derived block. The amplitude thresholds are applied before any discrimination to the signal to cutting horizontally the cardiogram signal to decrease the $\mathrm{P}$ and $\mathrm{T}$ wave's effect compared to the $\mathrm{R}$ wave. With a random distortion of the signal within the real world, it'll cause several false peak detections with high peak distortion, maximizing peak width and wave height and thus can be by pass this by homogenizing the derivative output of the signal. The homogeneity decides the status of the wave, the peak extent and therefore, the width of every peak with the interval specified. The signal is distinguished to supply information regarding the QRS-complex slope. The transfer function is:

$$
\mathrm{H}(\mathrm{z})=\frac{1}{10}\left(-2 \mathrm{z}^{-2}-\mathrm{z}^{-1}+\mathrm{z}^{1}+2\right)
$$

The difference version of this filter is:

$$
\mathrm{Y}(\mathrm{n})=\frac{1}{8}\left(-2 \mathrm{z}^{-2}-\mathrm{z}^{-1}+\mathrm{z}^{1}+2 \mathrm{z}^{2}\right)
$$

Squaring function: Following derivative, consecutive output signal is square. Become entire information positive, the derivative output is amplified nonlinear and also the QRS complex are assert as:

$$
\mathrm{Y}(\mathrm{NT})=[\mathrm{x}(\mathrm{NT})]^{2}
$$

Where:

(NT) : Input of ECG signal

$\mathrm{Y}(\mathrm{NT})$ : Squared of ECG input signal

Moving window integration: The output signal from the squaring process is entered to the moving-window integration block. The equation of the process is:

$$
y(n) \frac{1}{N}[x(n-(N-1)+x(n-(N-2))+, \ldots,+x(n)]
$$

Adaptive threshold: Following initial peak determination using threshold " $\mathrm{a}$ ' $(\mathrm{a}=$ mean (signal)), to determine threshold "a', using Eq. 6:

$$
\text { [Threshold }=\text { mean }(\text { Signal }) * 0.3]
$$

\section{RESULTS AND DISCUSSION}

Actual electrocardiogram signals originated from the MIT-BIH standard info were employed in the action. The MIT-BIH info include 48 record and half-hour with 2 recording channels for EKG (Llamedo and Martinez, 2011). All recordings are worked with a frequency of $360 \mathrm{~Hz}$ with 11-bit accuracy over range $(10 \mathrm{mV})$. The works have been done on MATLAB 2016b platform. The results divided into 2 parts, results of nonrecursive median filter and results of QRS detection as follows:

Non-recursive median filter output: Two stages non-recursive median filter has been applied to get rid of the wander drift from electrocardiogram signal. Median filter starts by choose any channel from electrocardiogram data say yi[n] have whole samples $\mathrm{N}$ for $\mathrm{N}=5000$ as illustrated in Fig. 3a. First step begin with filter window width is fs/2, value of input data yi[n] detected and keep in an array $\mathrm{xm} 1[\mathrm{n}]$. Using variable window size from fs/2 to fs/4 to work out and store values of input data yi[n] in an array $x m 1[n]$ from $\mathrm{N}$-fs $/ 4+1$ to $\mathrm{N}$ points. The next step median filter begin by using window width fs, then takes initial stage data $\mathrm{xm} 1[\mathrm{n}]$ having all samples ' $\mathrm{N}$ ' employing a variable window size of fs/ 2 to fs $/ 4$ to work out and store values of data $x \mathrm{~m} 1[\mathrm{n}]$ in array $\mathrm{xm} 2[\mathrm{n}]$ from 1 to fs/2 points, ultimately victimization variable window size of fs to fs/2 for input data xm1[n] to be specified and keep in array xm2[n] from $\mathrm{N}-\mathrm{fs} / 2+1$ to $\mathrm{N}$ points. Subtract second stage median filter output xm2[n] from input data $\mathrm{yi}[\mathrm{n}]$ for removing of baseline wander from signal $\mathrm{xf}[\mathrm{n}]$ use Eq. 7: 


$$
\mathrm{XR}[\mathrm{n}]=\mathrm{Yi}[\mathrm{n}]-\mathrm{Xm} 2[\mathrm{n}]
$$

Where:

$\mathrm{XR}[\mathrm{n}] \quad$ : The Baseline drift free signal

$\mathrm{Yi}[\mathrm{n}] \quad$ : The original signal

$\mathrm{Xm} 2[\mathrm{n}]$ : The output median signal after second median filter (fs)

Performance evaluation of the QRS detection: To estimate the performance of the proposed new
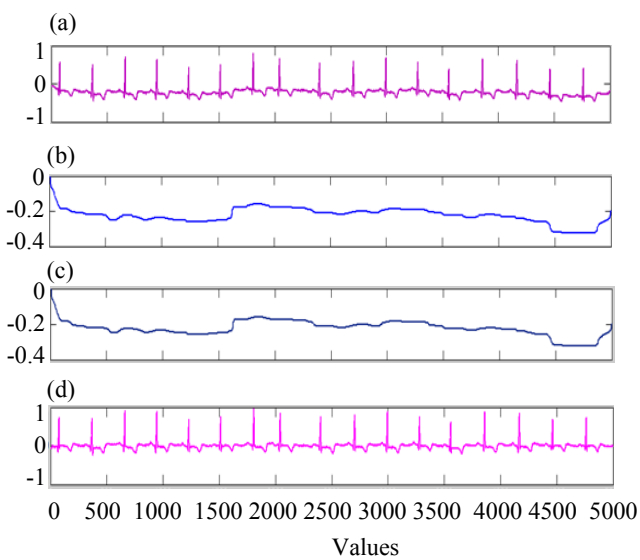

Fig. 3(a-d): Outputs of median filter, (a) Original signal yi[n]; ECG signal from channel: 2, (b) First step median filter output $\mathrm{xm} 1[\mathrm{n}]$; Output of the first median filter with window length = 181, (c) Second step median filter output $\mathrm{xm} 2[\mathrm{n}]$; Output of the second median filter with window length $=361$ and (d) Drift line free signal xf[n]; ECG signal-baseline drift methodology, this technique was performed with the MIT-BIH cardiac arrhythmia database (MIT-BIH AD). Different factors were tested that are Positive predictively $(+\mathrm{P})$, Sensitivity (Se) and Error rate (Er). In addition, other performance factor called F-score is also utilized in order to determining the efficiency of the current action, additionally to use of Accuracy (ACC) during this research. The achievement of the suggested methodology, in terms of Positive predictively $(+\mathrm{P})$, Sensitivity (Se), Accuracy (ACC), Error rate (Er) and F-score given in Eq. 8-12 (Rodriguez et al., 2014) (Table 2 and Fig. 4):

$$
\begin{gathered}
\mathrm{P}=\frac{\mathrm{TP}}{\mathrm{TP}+\mathrm{FP}} \\
\mathrm{Se}=\frac{\mathrm{TP}}{\mathrm{TP}+\mathrm{FN}} \\
\text { Acc }=\frac{\mathrm{TP}}{\mathrm{TP}+\mathrm{FP}+\mathrm{FN}} \\
\mathrm{Er}=\frac{\mathrm{TP}}{\mathrm{TP}+\mathrm{FP}} \\
\text { F-score }=\frac{(2 \times \mathrm{P} \times \mathrm{Se})}{\mathrm{P}+\mathrm{Se}}
\end{gathered}
$$

Where:

FN : Number of incomprehensible peaks

TP : Number of accurately detected peaks

FP : Number of false peaks

Table 2: Performance of the proposed work on ECG signals (30 min) of MIT-BIH arrhythmia database compared with Jenkal et al. (2015)

\begin{tabular}{llccclllll}
\hline $\begin{array}{l}\text { ECG } \\
\text { record }\end{array}$ & $\begin{array}{l}\text { Total } \\
\text { beats }\end{array}$ & $\begin{array}{c}\text { TP } \\
\text { (beats) }\end{array}$ & $\begin{array}{c}\text { FP } \\
\text { (beats) }\end{array}$ & $\begin{array}{c}\text { FN } \\
\text { (beats) }\end{array}$ & Se (\%) & + P(\%) & Acc (\%) & Er (\%) & F-score \\
\hline 100 & 2273 & 2273 & 0 & 0 & 100 & 100 & 100 & 0 & 1 \\
101 & 1865 & 1858 & 7 & 0 & 99.62607 & 100 & 99.62607 & 0.003753 & 0.998127 \\
102 & 2187 & 2187 & 0 & 1 & 100 & 99.9543 & 99.9543 & 0.000457 & 0.999771 \\
103 & 2084 & 2084 & 0 & 0 & 100 & 100 & 100 & 0 & 1 \\
104 & 2229 & 2229 & 0 & 7 & 100 & 99.68697 & 99.55337 & 0.004486 & 0.997762 \\
105 & 2572 & 2572 & 0 & 10 & 100 & 99.6127 & 99.6127 & 0.003888 & 0.99806 \\
106 & 2027 & 2024 & 3 & 0 & 99.85222 & 100 & 99.85222 & 0.00148 & 0.999261 \\
107 & 2137 & 2137 & 0 & 6 & 100 & 99.72002 & 99.72002 & 0.002808 & 0.998598 \\
108 & 1763 & 1756 & 10 & 0 & 99.43598 & 100 & 99.43598 & 0.005672 & 0.997172 \\
121 & 1863 & 1863 & 0 & 4 & 100 & 99.78575 & 99.78575 & 0.002147 & 0.998928 \\
122 & 2476 & 2476 & 0 & 0 & 100 & 100 & 100 & 0 & 1 \\
123 & 1518 & 1518 & 0 & 1 & 100 & 99.93417 & 99.93417 & 0.000659 & 0.999671 \\
124 & 1619 & 1617 & 2 & 0 & 99.87662 & 100 & 99.87662 & 0.001235 & 0.999383 \\
200 & 2601 & 2601 & 0 & 5 & 100 & 99.8081 & 99.617 & 0.003845 & 0.998081 \\
201 & 1963 & 1963 & 0 & 1 & 100 & 99.94908 & 99.94908 & 0.000509 & 0.999745 \\
202 & 2136 & 2136 & 0 & 0 & 100 & 100 & 100 & 0 \\
203 & 2980 & 2980 & 0 & 3 & 100 & 99.899 & 99.66555 & 0.003356 & 0.998325 \\
205 & 2656 & 2653 & 3 & 0 & 99.88705 & 100 & 99.88705 & 0.00113 & 0.999435 \\
207 & 1860 & 1858 & 0 & 10 & 100 & 99.46524 & 99.46524 & 0.005376 & 0.997319 \\
208 & 2955 & 2955 & 0 & 11 & 100 & 99.62913 & 99.49495 & 0.005076 & 0.997468 \\
209 & 3005 & 3005 & 0 & 19 & 100 & 99.37169 & 99.37169 & 0.006323 & 0.996849 \\
Average & 46769 & 46745 & 25 & 78 & 99.94 & 99.85 & 99.8 & 0.05 & 0.999 \\
\hline
\end{tabular}


Int. J. Syst. Signal Control Eng. Appl., 12 (1): 13-20, 2019
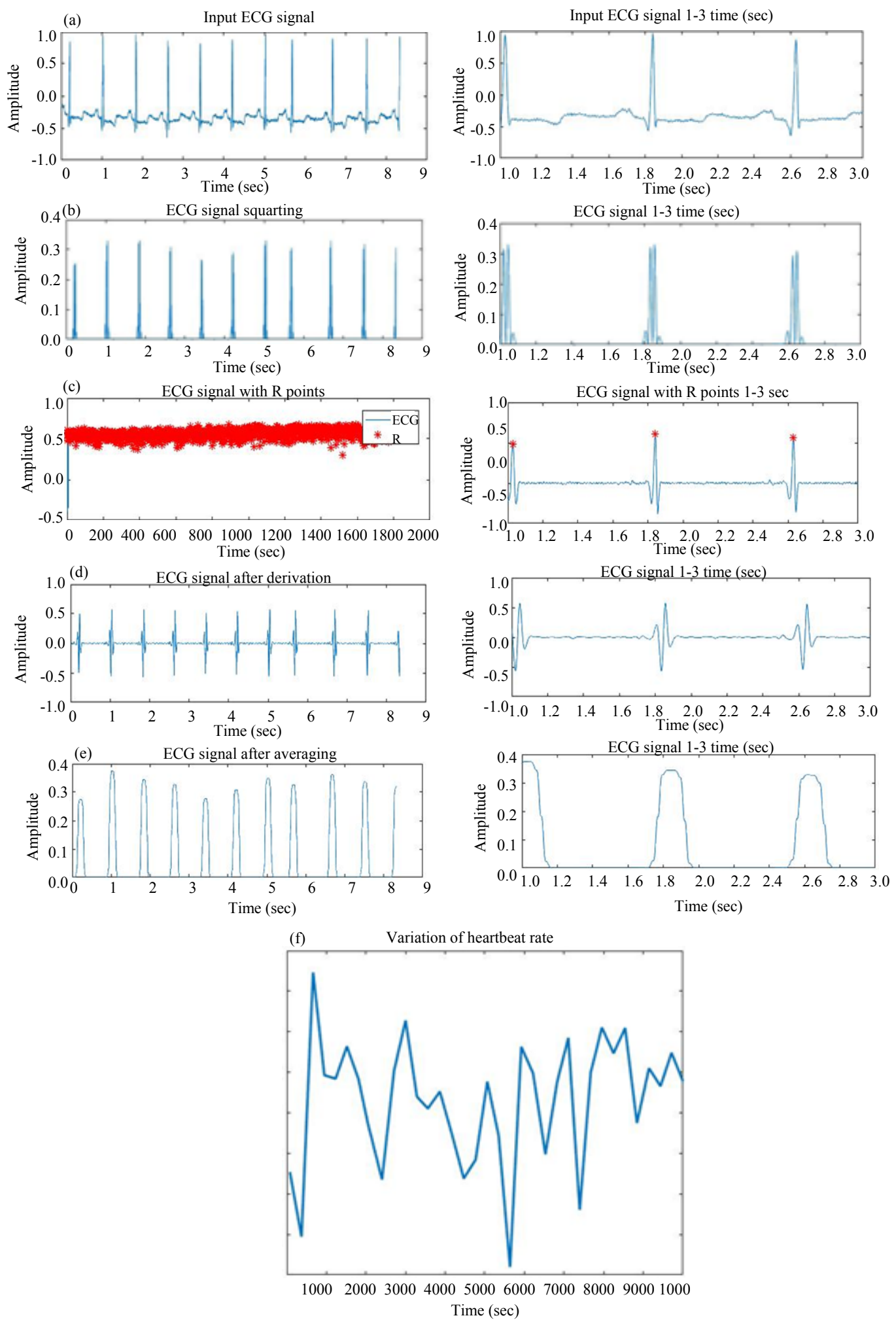

Fig. 4(a-f): ECG signal from record 100, (a) Original ECG (b) First derivative, (c) After squaring, (d) After moving average, (e) ECG signal with R point and (f) Variation of heartbeat rat 
Int. J. Syst. Signal Control Eng. Appl., 12 (1): 13-20, 2019

Table 3: Performance of the recent work on ECG signals of MIT-BIH arrhythmia database (Jenkal et al., 2015)

\begin{tabular}{|c|c|c|c|c|c|c|}
\hline ECG record & Total beats & TP (beats) & FP (beats) & FN (beats) & $\mathrm{Pp}(\%)$ & Se (\%) \\
\hline$\overline{100}$ & 2273 & 2273 & 0 & 0 & 100 & 100 \\
\hline 101 & 1865 & 1865 & 1 & 1 & 99.95 & 99.95 \\
\hline 102 & 2187 & 2188 & 1 & 0 & 99.95 & 100 \\
\hline 103 & 2084 & 2084 & 0 & 0 & 100 & 100 \\
\hline 104 & 2229 & 2230 & 1 & 0 & 99.96 & 100 \\
\hline 105 & 2572 & 2561 & 9 & 20 & 99.65 & 99.23 \\
\hline 106 & 2027 & 2021 & 0 & 6 & 100 & 99.7 \\
\hline 107 & 2137 & 2168 & 31 & 0 & 98.59 & 100 \\
\hline 108 & 1763 & 1774 & 17 & 6 & 99.05 & 99.66 \\
\hline 121 & 1963 & 1957 & 0 & 0 & 100 & 100 \\
\hline 122 & 2476 & 2476 & 0 & 0 & 100 & 100 \\
\hline 123 & 1518 & 1518 & 0 & 0 & 100 & 100 \\
\hline 124 & 1619 & 1619 & 0 & 3 & 100 & 99.81 \\
\hline 200 & 2601 & 2598 & 0 & 3 & 99.49 & 99.88 \\
\hline 201 & 1963 & 1957 & 10 & 16 & 100 & 99.19 \\
\hline 202 & 2136 & 2131 & 0 & 5 & 100 & 99.77 \\
\hline 203 & 2980 & 2920 & 0 & 60 & 100 & 97.99 \\
\hline 205 & 2656 & 2637 & 0 & 19 & 100 & 99.28 \\
\hline 207 & 1860 & 1858 & 5 & 7 & 99.73 & 99.62 \\
\hline 208 & 2955 & 2950 & 2 & 7 & 99.93 & 99.76 \\
\hline 209 & 3005 & 3005 & 0 & 0 & 100 & 100 \\
\hline Average & 46769 & 46769 & 77 & 153 & 99.84 & 99.67 \\
\hline
\end{tabular}

Table 4: Recent QRS detection methods compared our algorithm

\begin{tabular}{llll}
\hline Methods & Se (\%) & Pp (\%) & Computational load \\
\hline Proposed methods & 99.94 & 99.85 & Low \\
Yeh and Wang (2008) & 99.85 & 99.95 & Low \\
Pan and Tompkins (1985) & 99.75 & 99.54 & High \\
Hamilton and Tompkins (1986) & 99.69 & 99.77 & Medium \\
Adnane et al. (2009) & 99.77 & 99.64 & Low \\
Sahambi et al. (1997) & 99.90 & 99.90 & Medium \\
Saxena et al. $(2002)$ & 98.80 & 99.86 & High \\
Ghaffari et al. (2008) & 99.89 & 99.71 & Low \\
Chouakri et al. (2011) & 99.91 & 99.81 & High \\
Rodriguez et al. $(2014)$ & 99.71 & 99.28 & High \\
Zidelmal et al. $(2014)$ & 99.84 & 99.91 & Low \\
\hline
\end{tabular}

The MIT-BIH cardiac arrhythmia database was examined during this experiment, besides together with baseline deviation it contains LBBB, RBBB, PVC and APC. Our QRS detection algorithmic program performs with 48 records fully length during this study. With removing of wander baseline drift, power noise and artifacts are removed too. MIT/BIH cardiac arrhythmia data record 100 contain noises with extremely baseline drifts that is clearly detected and shown in Fig. 4. The effectiveness of the new suggested technique emulated and evaluated in contrast to recent work (Jenkal et al., 2015) in contrast to alternative researchers wherever they largely rated the effectiveness of QRS detector technique utilizing MIT/BIH cardiac rhythm irregularities data-base. Performance of proposed new system has well-tried to be superior to the recent papers (Jenkal et al., 2015) as shown in Table 1 and 2 . We have a tendency to signify that achievement average of all 48 records and compared to alternative previous strategies in Table 3. Our proposed new technique offered Sensitivity (Se\%) and Positive predictively $(+\mathrm{P})$ of cardiac rhythm irregularities data-base as 99.94 and $99.85 \%$, severally, that is higher compared to alternative ways (Table 4).

\section{CONCLUSION}

An active and dependable QRS complex determination technique primarily centered-median filter and signal reshaping was instructed. The proposed method has been tested on standard MIT/BIH data base and conjointly applied on real graphical record patients. The median filter approach is employed to address the base line from the electrocardiogram signal and so an automatic R-peaks detection method with sequence steps was instructed to detect the QRS position. The proposed methodology offered high QRS detection performance compared to recent algorithms. supported the findings, a low detection error rate of $0.05 \%$, Positive predictivity $(\mathrm{P}+)$ of $99.85 \%$, Sensitivity (Se) of $99.94 \%$ and an average F-score of 0.999 are determined for the proposed electrocardiogram detector that the most effective among previous algorithms.

\section{REFERENCES}

Adnane, M., Z. Jiang and S. Choi, 2009. Development of QRS detection algorithm designed for wearable cardiorespiratory system. Comput. Methods Prog. Biomed., 93: 20-31. 
Arzeno, N.M., Z.D. Deng and C.S. Poon, 2008. Analysis of first-derivative based QRS detection algorithms. IEEE Trans. Biomed. Eng., 55: 478-484.

Benitez, D.S., P.A. Gaydecki, A. Zaidi and A.P. Fitzpatrick, 2000. A new QRS detection algorithm based on the hilbert transform. Comput. Cardiol., 27: 379-382.

Chen, S.W., H.C. Chen and H.L. Chan, 2006. A real-time QRS detection method based on moving-averaging incorporating with wavelet denoising. Comput. Methods Programs Biomed., 82: 187-195.

Chouakri, S.A., F. Bereksi-Reguig and A. Taleb-Ahmed, 2011. QRS complex detection based on multi wavelet packet decomposition. Applied Math. Comput., 217: 9508-9525.

Christov, I.I., 2004. Real time electrocardiogram QRS detection using combined adaptive threshold. Biomed. Eng. Online, Vol. 3, 10.1186/1475-925X-3-28

Dilaveris, P.E., E.J. Gialafos, S.K. Sideris, A.M. Theopistou and G.K. Andrikopoulos et al., 1998. Simple electrocardiographic markers for the prediction of paroxysmal idiopathic atrial fibrillation. Am. Heart J., 135: 733-738.

Dohare, A.K., V. Kumar and R. Kumar, 2014. An efficient new method for the detection of QRS in electrocardiogram. Comput. Electr. Eng., 40: 1717-1730.

El-Dahshan, E.S.A., 2011. Genetic algorithm and wavelet hybrid scheme for ECG signal denoising. Telecommun. Syst., 46: 209-215.

Ghaffari, A., H. Golbayani and M. Ghasemi, 2008. A new mathematical based QRS detector using continuous wavelet transform. Comput. Electr. Eng., 34: 81-91.

Ghaffari, A., M.R. Homaeinezhad, M. Akraminia, M. Atarod and M. Daevaeiha, 2009. A robust wavelet-based multi-lead electrocardiogram delineation algorithm. Med. Eng. Phys., 31: 1219-1227.

Hamilton, P.S. and W.J. Tompkins, 1986. Quantitative investigation of QRS detection rules using the MIT/BIH arrhythmia database. IEEE. Trans. Biomed. Eng., 33: 1157-1165.

Huang, T.S., 1981. Two-Dimensional Digital Signal Processing I: Linear Filters. 1st Edn., Springer, New York, USA., ISBN-13: 978-3540103486,.

Jenkal, W., R. Latif, A. Toumanari, A. Dliou and O. El B'charri, 2015. Enhanced algorithm for QRS detection using Discrete Wavelet Transform (DWT). Proceedings of the 2015 27th International Conference on Microelectronics (ICM'15), December 20-23, 2015, IEEE, Casablanca, Morocco, pp: 39-42.
Li, H. and J. Tan, 2006. Body sensor network based context aware QRS detection. Proceedings of the 2006 International Conference on Pervasive Health and Workshops, November 29-December 1, 2006, IEEE, Innsbruck, Austria, pp: 1-8.

Llamedo, M. and J.P. Martinez, 2011. Heartbeat classification using feature selection driven by database generalization criteria. IEEE Trans. Biomed. Eng., 58: 616-625.

Manikandan, M.S. and K.P. Soman, 2012. A novel method for detecting R-peaks in electrocardiogram (ECG) signal. Biomed. Signal Process. Control, 7: 118-128.

Martinez, J.P., R. Almeida, S. Olmos, A.P. Rocha and P. Laguna, 2004. A wavelet-based ECG delineator: Evaluation on standard databases. IEEE. Trans. Biomed. Eng., 51: 570-581.

Mehta, S.S. and N.S. Lingayat, 2008. SVM-based algorithm for recognition of QRS complexes in electrocardiogram. IRBM., 29: 310-317.

Pan, J. and W.J. Tompkins, 1985. A real-time QRS detection algorithm. IEEE Trans. Biomed. Eng., 32: 230-236.

Plesnik, E., O. Malgina, J.F. Tasic and M. Zajc, 2012. Detection of the electrocardiogram fiducial points in the phase space using the euclidian distance measure. Med. Eng. Phys., 34: 524-529.

Rodriguez, R., A. Mexicano, J. Bila, R. Ponce and S. Cervantes, 2014. Adaptive threshold and principal component analysis for features extraction of electrocardiogram signals. Proceedings of the 2014 International Symposium on Computer, Consumer and Control, June 10-12, 2014, IEEE, Taichung, Taiwan, pp: 1253-1258.

Sahambi, J.S., S.N. Tandon and R.K.P. Bhatt, 1997. Using wavelet transforms for ECG characterization: An on-line digital signal processing system. IEEE. Eng. Med. Biol. Mag., 16: 77-83.

Saxena, S.C., V. Kumar and V. Hamden, 2002. Feature extraction from ECG signals using wavelet transforms for disease diagnostics. Int. J. Syst. Sci., 33: 1073-1085.

Silva, I., G.B. Moody and L. Celi, 2011. Improving the quality of ECGs collected using mobile phones: The physionet/computing in cardiology challenge 2011. Proceedings of the 2011 International Conference on Computing in Cardiology, September 18-21, 2011, IEEE, Hangzhou, China, pp: 273-276.

Sunkaria, R.K., S.C. Saxena, V. Kumar and A.M. Singhal, 2010. Wavelet based R-peak detection for heart rate variability studies. J. Med. Eng. Technol., 34: 108-115.

Vijaya, G., V. Kumar and H.K. Verma, 1997. Artificial neural network based wave complex detection in electrocardiograms. Int. J. Syst. Sci., 28: 125-132. 
WHO., 2011. Global Status Report on Alcohol and Health. World Health Organization, Geneva, Switzerland, ISBN-13: 9789241564151, Pages: 286.

Yeh, Y.C. and W.J. Wang, 2008. QRS complexes detection for ECG signal: The difference operation method. Comput. Methods Programs Biomed., 91: 245-254.
Zhang, F. and Y. Lian, 2009. QRS detection based on multiscale mathematical morphology for wearable ECG devices in body area networks. IEEE. Trans. Biomed. Circuits Syst., 3: 220-228.

Zidelmal, Z., A. Amirou, D. Ould-Abdeslam, A. Moukadem and A. Dieterlen, 2014. QRS detection using S-Transform and Shannon energy. Comput. Methods Programs Biomed., 116: 1-9. 